\title{
Comparative analysis of human sperm glycocalyx from different freezability ejaculates by lectin microarray and identification of $A B A$ as sperm freezability biomarker
}

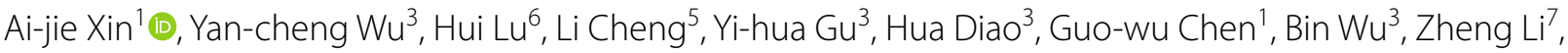 \\ Sheng-ce Tao ${ }^{5}$, Xiao-xi Sun ${ }^{1,2^{*}}$ and Hui-juan Shi ${ }^{3,4^{*}}$
}

\begin{abstract}
Background: Semen cryopreservation has been widely applied in assisted reproductive technologies and sperm bank, but it causes considerable impairments on sperm quality. It is necessary to find an evaluation indicator for determining the sperm-freezing tolerance.

Methods: The glycocalyx of good freezability ejaculates was compared with poor freezability ejaculates by lectin microarray. The significant different lectins were validated by flow cytometry (FACS). To analyze the relationship between the potential biomarker and the tolerance of sperm to cryopreservation, 60 samples with different recovery rates were collected and detected the lectin-binding intensity by FACS. The receiver operating characteristic (ROC) curve was analyzed to test the capability of the lectin as a potential biomarker for detecting the sperm freezablility.

Results: $A B A$ and DSL were found to develop significant differences between them. Further validation showed that ABA was significantly negative correlated with the sperm recovery rates $(r=-0.618, P<0.000)$ and could be a potential biomarker for predicting sperm freezability $(A \cup C=0.733 \pm 0.067,95 \% \mathrm{Cl} 0.601-0.865, P<0.01)$.
\end{abstract}

Conclusion: ABA could be a potential biomarker for predicting sperm freezability. It will help to reduce sperm-freezing recovery tests and improve the efficiency of cryopreservation in human sperm bank.

Keywords: Cryopreservation, Freezability, Lecin microarray, Human sperm, Glycocalyx

\section{Background}

After decades of development, semen cryopreservation has been widely applied in assisted reproductive technologies (ART) and sperm bank [1,2]. The technology helps men with azoospermia or severe hereditary disease related with infertility have the opportunity to have children using the donor's sperm from sperm bank by in vitro fertilization (IVF) or intracytoplasmic sperm

\footnotetext{
*Correspondence: xiaoxi_sun@aliyun.com; shihuijuan2011@163.com ${ }^{2}$ Key Laboratory of Female Reproductive Endocrine Related Disease, Obstetrics and Gynecology Hospital, Fudan University, Shanghai 200011, China

${ }^{4}$ Shanghai Key Laboratory of Reproductive Medicine, Shanghai Jiao Tong University School of Medicine, Shanghai 200025, China

Full list of author information is available at the end of the article
}

injection (ICSI). In addition, for some patients who are about to undergo chemotherapy treatment or other events associated with loss of fertility, sperm cryopreservation can preserve their fertility and make them have their own children later $[3,4]$. However, cryopreservation caused dramatic impairments to sperm containing sperm motility, viability, DNA integrity, plasma membrane, and matrix density of mitochondrial, and reduced the sperm ability of penetration of cervical mucus and egg [5-9]. The improvement of the cryopreservation technology and the stability of the recovery rate directly impact on pregnancy rate.

In order to ensure the safe and effective supply of semen, the better quality, higher recovery rate of semen is the working focus of sperm bank. However, the tolerance

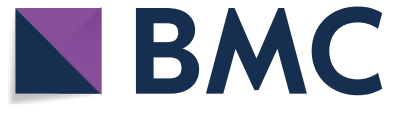

(c) The Author(s) 2018. This article is distributed under the terms of the Creative Commons Attribution 4.0 International License (http://creativecommons.org/licenses/by/4.0/), which permits unrestricted use, distribution, and reproduction in any medium, provided you give appropriate credit to the original author(s) and the source, provide a link to the Creative Commons license, and indicate if changes were made. The Creative Commons Public Domain Dedication waiver (http://creativecommons.org/ publicdomain/zero/1.0/) applies to the data made available in this article, unless otherwise stated. 
of sperm to cryopreservation varies in individuals. There are always some semen samples presenting poor ability to resist cryopreservation in clinic. Until now, many studies on human semen have attempted to find biomarkers of freezability. But, some of them showed controversial. Prefreezed motility has been reported to be correlated with cryosurvival rate $[10,11]$. High initial motility and sperm density result in high recovery rate [12]. While other studies reported that the parameters of conventional semen analysis including sperm concentration, motility, WHO morphology and total motile count showed no correlation with the sperm motility recovery rates [13-15]. These suggested that the conventional semen parameters have no sufficient capacity to predict the sperm freezability. In addition, the traditionary method by post-thawed recovery is not only time-consuming, but also labor and reagents wasting, which makes an evaluation indicator for determining the sperm freezing tolerance urgent.

The sperm glycocalyx is a dense carbohydrate layer with $20-60 \mathrm{~nm}$ thick, coating on the sperm membrane outmost surface with protein and lipid [16]. It plays an important role in sperm maturation, motility and fertilization [16-18]. During the process of sperm formation, maturation, capacitation and acrosome reaction, the glycoprotein on the sperm surface is largely rearranged. Subtle change in glycocalyx has significant effect on sperm fertility [17, 19-22]. Reports about the cryodamage of sperm glycocalyx are few due to the technical limitation. Only several papers about the avian sperm carbohydrate changes caused by cryopreservation. And its alterations were associated with the impaired fertility $[23,24]$. As a group of natural glycan binders, lectins labeled with different conjugates can detect individual glycans by immunocytochemistry, immunohistochemistry or flow cytome. This is the main method to study the composition of cell glycocalyx. Recently, our lab reported a sensitive and high-through technology-lectin microarray to analyze the sperm glycocalyx $[22,25]$. It accelerated the study on sperm glycocalyx.

Therefore, the aim of this study was to find the biomarkers related with human sperm freezability through comparing the glycocalyx between good freezability ejaculates (GFEs) and poor freezability ejaculates (PFEs) by lectin microarray. These will be conducive to optimization of sperm cryopreservation methods, screening of the high quality sperm and improvement of the sperm fertilization.

\section{Methods}

\section{Sperm collection}

All the semen samples in this study were collected in Human Sperm Bank of Renji Hospital, Shanghai Jiao
Tong University School of Medicine. The age of the donors ranged from 20 to 35 years. The donors were instructed to collect semen samples through masturbation after 3-5 days sexual abstinence. Semen was harvested in sterile containers. All these samples were evaluated for volume, sperm concentration, total motility, progressive motility (PR), and non-progressive motility (NP) according to the fifth edition of WHO laboratory manual. The samples with normal semen parameters were included; i.e., they presented the normal volume $(\geq 2 \mathrm{ml})$, concentration $\left(\geq 15 \times 10^{6} / \mathrm{ml}\right)$ and total motility $(\geq 40 \%)$.

Liquefied semen samples were divided into two aliquots. One of the aliquots was performed to cryopreservation. The other aliquot was directly fixed for lectin microarray analysis or flow cytometry.

All the donors have given the written informed consent. This research was approved by the Institutional Review Committee of Shanghai Jiao Tong University. All experiments were performed in accordance with the relevant guidelines and regulations.

\section{Semen cryopreservation and thawing}

The semen samples were cryopreserved by direct vapor nitrogen freezing method [26]. The liquefied semen samples were mixed with an equal volume of CryoSperm ${ }^{\mathrm{TM}}$ (ORIGIO, USA), followed with incubation at room temperature (RT) for $10 \mathrm{~min}$. Then, the equilibrated samples were transferred to cryovials and placed about $10 \mathrm{~cm}$ top from the surface of liquid nitrogen. After incubated for $20 \mathrm{~h}$, the cryovials were preserved in liquid nitrogen at $-196^{\circ} \mathrm{C}$. After $24 \mathrm{~h}$, the cryovials were immediately moved from liquid nitrogen into water bath at $37{ }^{\circ} \mathrm{C}$ for $5 \mathrm{~min}$. The sperm total motility was examined to calculate the recovery rate. The recovery rate $(\%)=$ Sperm motility after cryopreservation/Sperm motility before cryopreservation $\times 100 \%$.

\section{Sperm preparation and lectin microarray analysis}

The sperm samples were prepared for lectin microarray analysis as our previously described [22, 25]. The fresh semen and the frozen-thawed semen were centrifuged (500 $\mathrm{g} \times 10 \mathrm{~min}$ ) for collecting the sperm cells and washed with PBS, followed with fixation with $2 \%$ paraformaldehyde containing $0.2 \%$ glutaraldehyde for $15 \mathrm{~min}$, and then wash with PBS once, before stored at $4{ }^{\circ} \mathrm{C}$ for the subsequent lectin microarray and flow cytometry experiments.

The preparation of lectin microarray was consistent with our previously reported [22, 25, 27]. Simply, 91 lectins with $1 \mu \mathrm{g} / \mu \mathrm{l}$ concentration were printed in triplicate on OPPolymer Slide H slides (CapitalBio, Beijing, China). Each slide contained 12 blocks with a matrix with $18 \times 16$ 
arrangement. Then, stored at $4{ }^{\circ} \mathrm{C}$ overnight for the lectins coated on the surface, the slides were ready for the sperm detection.

Lectin microarray was firstly blocked in $10 \mathrm{mM}$ Tris Buffered Saline with 0.5\% (v/v) Tween-20 (TBST) for $1 \mathrm{~h}$ at RT and then washed once in PBST for $10 \mathrm{~min}$, followed with twice in PBS for $10 \mathrm{~min}$. The fixed sperm labeled with propidine iodide (PI, $20 \mu \mathrm{g} / \mathrm{ml}$ ) was adjusted concentration to $5 \times 10^{6}$ spermatozoa in $200 \mu \mathrm{l}$ PBS with $50 \mu \mathrm{M} \mathrm{CaCl}_{2}$ and $50 \mu \mathrm{M} \mathrm{MnCl}_{2}$ for each block of lectin microarray, and then incubated in a wet box for $1 \mathrm{~h}$ at RT in the dark. Each sample was repeated four times in and between slides with a diagonal manner. After the excess and unbound spermatozoa gently removed by submerging and inverting the slides in PBST, the air-dried slides were scanned with a GenePix 4200A (Molecular Devices, Sunnyvale, CA) at $5 \mu \mathrm{m}$ resolution with the scanning condition set to $532 \mathrm{~nm}$ filter and 40\% PMT value.

\section{Validation by flow cytometry}

Sixty samples with different recovery rates were collected and fixed. The seminal parameters and recovery rate of the samples were in Additional file $1.2 \times 10^{6}$ spermatozoa were re-suspended with $90 \mu \mathrm{l} \mathrm{PBS}$ and added $10 \mu \mathrm{l}$ fluorescein isothiocyanate (FITC)-labeled lectin with the final concentration $100 \mu \mathrm{g} / \mathrm{ml}$, then incubated for $30 \mathrm{~min}$ at $37^{\circ} \mathrm{C}$ in the dark. After that the spermatozoa were washed once and re-suspended with $500 \mu \mathrm{l}$ PBS, to be analyzed in a Facs Calibur Flow cytometer using WinMID2.9 software.

\section{Statistical analysis}

The binding signals of the sperm with lectin microarray were extracted by GenePix pro 6.0. The signal intensity to the local background noise ratio (SNR) was defined as F532 Mean/B532 Mean, and all the spots' SNRs of lectin microarray were calculated and normalized. Because of each sample performed four blocks repetition and each lectin having three repeats on each block, each sample had 12 SNRs. All the lectin binding signal data of GFEs and PFEs were averaged, respectively. The cut off value of the positive lectin binding was set as $\mathrm{SNR} \geq 2$.

Data analysis and graphs were conducted by SPSS 20.0 and GraphPad Prism 5 and all the data was described as the mean \pm SEM. The significantly different SNRs and the Geo Mean between the GFEs and the PFEs samples were determined by Independent-Samples T Test. The correlation of ABA and recovery rate was analyzed via Pearson Correlations and Linear Regression. ROC analyses were performed with the ABA binding signal intensity plotted against the PFEs. The area under the ROC curves (AUC) were calculated to evaluate the sperm freezability.
Table 1 The characteristics of the GFEs and the PFEs

\begin{tabular}{lccc}
\hline & GFEs $(\mathbf{n}=6)$ & PFEs $(\mathbf{n}=6)$ & $P$ \\
\hline Age & $26.33 \pm 1.82$ & $25.50 \pm 1.15$ & 0.707 \\
Semen volume (ml) & $3.53 \pm 0.62$ & $4.57 \pm 0.20$ & 0.145 \\
Sperm concentration $\left(10^{6}\right.$ per $\left.\mathrm{ml}\right)$ & $85.67 \pm 12.66$ & $73.67 \pm 12.30$ & 0.512 \\
Total motility (PR+ NP, \%) & $57.38 \pm 3.92$ & $53.75 \pm 3.18$ & 0.489 \\
Recovery rate (\%) & $58.13 \pm 3.00$ & $25.60 \pm 1.34$ & 0.000 \\
\hline
\end{tabular}

All values are mean \pm SEM

GFEs good freezability ejaculates, PFEs poor freezability ejaculates, $P R$ progressive motility, NP non-progressive motility

\section{Results}

$A B A$ and $D S L$ being related with sperm freezabiltiy In order to compare the lectin binding profilings between GFEs and PFEs, we collected 12 semen samples (GFEs, $\mathrm{n}=6$; PFEs, $\mathrm{n}=6$ ) according to the recovery rate. Among them, semen with the recovery rate more than $50 \%$ were classified to the GFEs group, while that of less than $30 \%$ were classified PFEs. As showed in Table 1, the average age of the enrolled donors was about 26 years, and semen parameters before cryopreservation had no significant difference between the two groups. Obviously, the recovery rate showed statistically different $(P<0.000)$. The GFEs and PFEs samples before cryopreservation were prepared and analyzed by lectin microarray. Through comparation of 91 lectins binding signal intensity between the two groups, Agaricus bisporusagglutinin (ABA) and Datura Stramonium Lectin (DSL) presented significant difference (Fig. 1). The ABA binding signal intensity of the GFEs showed lower than that of PFEs, while the DSL binding signal intensity was opposite, it was higher in the GFEs.

\section{Validation of $A B A$ and DSL by FACS}

To validate the different lectins binding between GFEs and PFEs, we used fluorescein isothiocyanate (FITC)labeled ABA and DSL to analyze the binding signal of sperm by flow cytometry (FACS). As indicated in Fig. 2, the fluorescence intensity of ABA in PFEs was significant increased than GEFs, which was totally consistent with the result of lectin microarray. However, the binding signal of DSL demonstrated no significant difference between the two groups. So, ABA was screened for the further experiments.

\section{ABA being a potential biomarker for detecting the sperm freezability}

To analyze the relationship between $\mathrm{ABA}$ and the tolerance of sperm to cryopreservation, sixty samples with different recovery rates were collected and detected the ABA binding intensity by FACS. The Pearson Correlation 


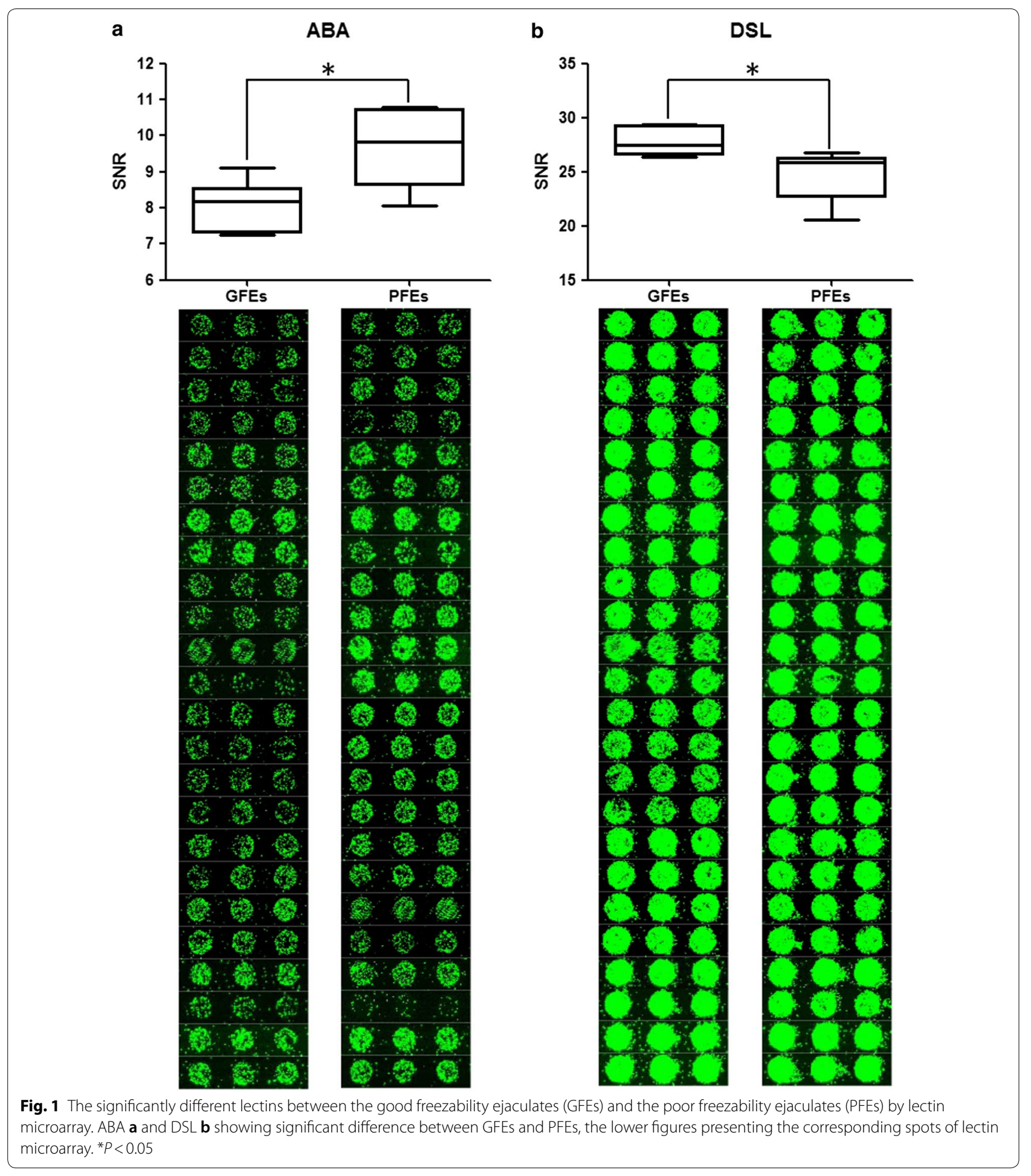

Coefficients demonstrated the binding intensity with ABA was significantly negative correlated with the sperm recovery rate $(r=-0.618, P<0.000)$. In addition, the linear regression relationship between them is as: $y=106.042-0.334 x$ (Fig. 3).
Furthermore, to test the capability of ABA as a potential biomarker for detecting the sperm freezability, Receiver Operating Characteristic (ROC) curve was analyzed. The recovery rate of $50 \%$ was set as the cut-off value of the tolerance of sperm to cryopreservation. As showed 
a

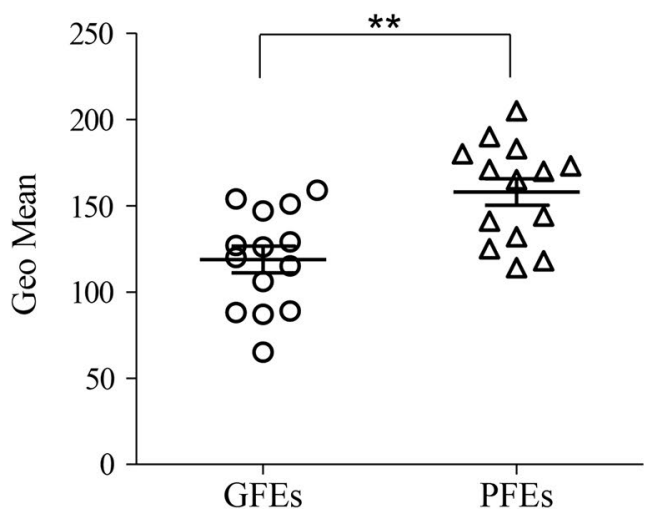

b

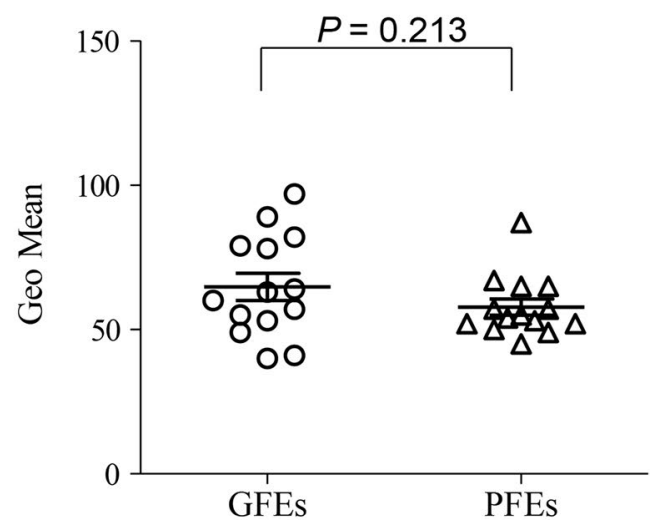

Fig. 2 Validation of sperm-lectin binding by flow cytometry. FACS analysis of the good freezability ejaculates (GFEs) and the poor freezability ejaculates (PFEs) labeled with FITC-ABA a or FITC-DLS b. ABA showing significant difference consistent with the results by lectin microarray; while DLS showing no statistical difference. ${ }^{* *} P<0.01$

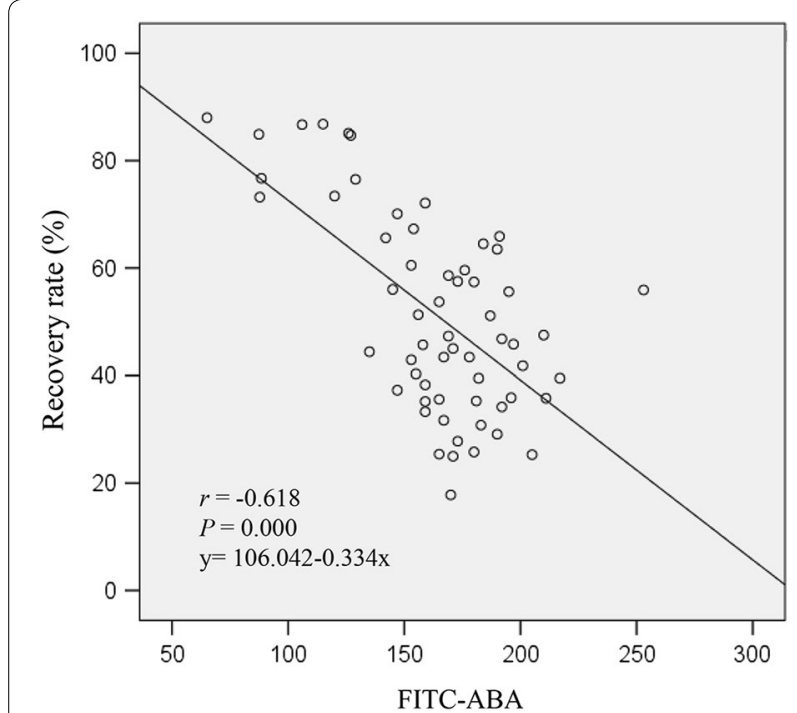

Fig. 3 Correlation of ABA and the recovery rate of human sperm. ABA was significantly negative correlated with the sperm recovery rates

in Fig. 4, the area under the curves (AUC) of ABA was $0.733 \pm 0.067$ (95\% CI $0.601-0.865, P<0.01)$, which indicated that ABA could serve as a potential biomarker for detecting the sperm freezability. The cut-off value of ABA based on the data was 157 with $57.1 \%$ specificity (95\% CI $0.372-0.755)$ and $87.5 \%$ sensitivity (95\% CI $0.710-0.965)$.

\section{Discussion}

High quality semen is the core and key of sperm bank. However, there are often some semen samples have better semen parameters before freezing, but poor and

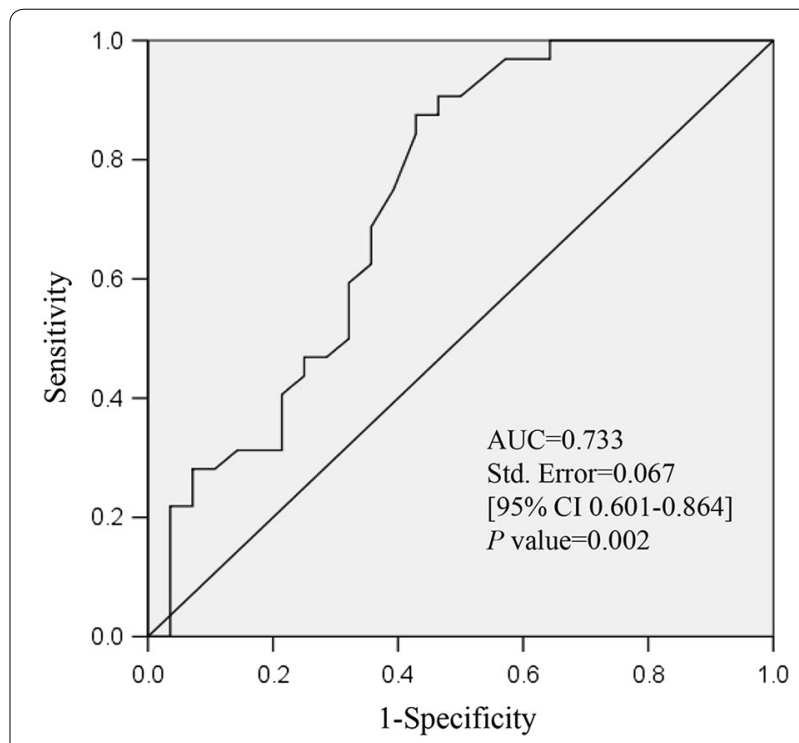

Fig. 4 Evaluation of ABA being biomarker for sperm freezability. The good freezability ejaculates as the control group $(n=32)$ and the poor freezability ejaculates as abnormal group $(n=28)$. The ROC curves and the corresponding AUCs were calculated by SPSS16.0

unqualified motility after cryopreservation. Prediction of sperm freezability and classification of human semen into GFEs or PFEs before cryopreservation will help to save time, money and labor and facilitate the full use of frozen-thawed human spermatozoa. In this study, by comparing the lectin binding profilings of sperm samples with high recovery rate (classified to GFEs) with that of sperm with low recovery rate (classified to PFEs) through the most comprehensive lectin microarray technology, 
$\mathrm{ABA}$ and DSL showed significant differences, and $\mathrm{ABA}$ had the biomarker potential for detecting the sperm freezability.

Sperm glycocalyx, composed of glycoproteins and glycolipids, located on the outer surface of the plasma membrane, protects sperm from the immune surveillance in the uterus and helps maintain sperm survival [28-30]. It is reported that cryopreservation changes the sperm carbohydrates in avian, and that is associated with the impaired fertility [23, 24]. In addition, we found that cryopreservation significantly changed the sperm glycocalyx in human, and the sialic acid, generally located at the terminal of the sugar chain of glycoprotein, was seriously lost [31]. This suggested to some extent that the glycocalyx played an important role on protecting sperm from cryopreservation. Studies of the effects of cryopreservation on spermatozoa mainly focused on proteomics of seminal plasma or sperm membranes in many mammal species [32-36]. To the best of our knowledge, this is the first study that associate the glycocalyx with semen freezabiltiy in human.

It is reported that Kruger strict morphology is significantly correlated with the progressive motility recovery rate $(r=0.294, P=0.028)$ and marginally significant with the relationship between cryosurvival rate $(r=0.249$, $P=0.064)$ [15]. While we found that the Pearson Correlation Coefficients of $\mathrm{ABA}$ and sperm recovery rates $(r=-0.618, P<0.000)$ was more relevant and significant than Kruger morphology. Jiang et al. [37] reported a multivariate model for predicting semen cryopreservation outcomes by three semen parameters, including progressive motility (PR), straight-line velocity (VSL) and average path velocity (VAP), and the AUC of the multivariate model is 0.789. In this study, the AUC of ABA was 0.733. It was illustrated that the one factor of ABA had considerable predictive capacity compared with the multivariate model.

According to the glycosylation site of the peptide chain, glycoproteins possess two type glycans, N-linked glycans and O-linked glycans. N-acetylgalactosamine (GalNAc) is generally added to serine (Ser) or threonine (Thr) residues at the first step in O-glycosylation of proteins by polypeptide $\mathrm{N}$-acetylgalactosaminyl transferase-6 (ppGalNAc-T6) catalyzation [38, 39], followed by galactose (Gal) and N-Acetylglucosamine (GlcNAc) transferation and added sialic acid (Sia) at the terminal of glycan chains. The lectin ABA specifically recognized the oligosaccharides of O-linked glycosylation (GalNAc-Ser/Thr) which was generally located in the inside of glycocalyx. In this study, the binding signal of ABA was significantly increased in PFEs. The sperm glycocalyx coated on the outmost surface of sperm membrane and played an important role on protecting sperm. The increase of ABA might be due to the imperfect glycocalyx and exposure of the inner oligosaccharides in PFEs. In addition, the previous paper in our lab had been found that the cryodamaged sperm showed the higher binding intensity to ABA than the fresh sperm [31]. It is suggested that the glycocalyx of PEFs had been impaired when ejaculated, and had no competence to resist cryopreservation.

It is reported that the protein composition has significant difference between GFEs and PFEs, and related with sperm motility and fertility [36, 37]. In this study, the sperm glycocalyx between them also showed significant difference. It is reported that the alteration of spermatozoal glycocalyx is associated with impaired fertility in the fowl [40]. It seems reasonable to hypothesize that sperm freezability is the inherent characteristic of sperm, and it varies in individuals. Furthermore, on the basis of mastering the technique of sperm cryopreservation and the quality control in laboratory, sperm freezability should be considered as one of the evaluation indicators of sperm quality. By comparing the glycocalyx between GFEs and PFEs prior to freezing, ABA will be one of potential biomarkers to predict the sperm freezability. This will help to reduce sperm freezing recovery tests, thereby reducing the workload and semen waste and improve the efficiency of cryopreservation in human sperm bank.

\section{Additional file}

Additional file 1. The characteristics of the sixty samples with different recovery rates.

\section{Authors' contributions}

HS and XS conceived and designed the experiments. AX performed the experiments and drafted the manuscript. YW and LC prepared the lectin microarry. GC, BW, HL and ZL provided clinical samples. ST, HD and YG involved in data analysis and provided technical assistance. All authors read and approved the final manuscript.

\section{Author details \\ ${ }^{1}$ Shanghai Ji Ai Genetics and IVF Institute, Obstetrics and Gynecology Hos- pital, Fudan University, Shanghai 200011, China. ${ }^{2}$ Key Laboratory of Female Reproductive Endocrine Related Disease, Obstetrics and Gynecology Hospital, Fudan University, Shanghai 200011, China. ${ }^{3}$ China National Population and Family Planning Key Laboratory of Contraceptive Drugs and Devices, SIPPR, Shanghai 200032, China. ${ }^{4}$ Shanghai Key Laboratory of Reproduc- tive Medicine, Shanghai Jiao Tong University School of Medicine, Shang- hai 200025, China. ${ }^{5}$ Shanghai Center for Systems Biomedicine, Key Laboratory of Systems Biomedicine (Ministry of Education), Shanghai Jiao Tong University, Shanghai 200240, China. ${ }^{6}$ Department of Allergy, Renji Hospital, Shanghai Jiao Tong University School of Medicine, Shanghai 200001, China. ${ }^{7}$ Department of Andrology and PFD, Center for Men's Health, Institute of Urology, Urologic Medical Center, Shanghai General Hospital, Shanghai Key Lab of Reproductive Medicine, Shanghai Jiao Tong University, Shanghai 200080, China.}

\section{Competing interests}

The authors declare that they have no competing interests.

\section{Availability of data and materials}

The datasets used and/or analyzed during the current study are available from the corresponding author on reasonable request. 


\section{Consent for publication}

The authors declare that they have no competing interests.

\section{Ethics approval and consent to participate}

All the donors have given the written informed consent. This research was approved by the Institutional Review Committee of Shanghai Jiao Tong University. All experiments were performed in accordance with the relevant guidelines and regulations.

\section{Funding}

This work was supported by the National Natural Science Foundation of China (Nos. 81401252 and 81270744), the Special Fund for Clinical research of Chinese Medical Association (No. 16020470663), the Foundation of Shanghai Municipal Commission of Health and Family Planning (No. 20164Y0157), the Foundation of Science and Technology Commission of Shanghai Municipality (No. 17JC1400902), the Foundation of Shanghai Ji Ai Genetics and IVF Institute (JA-2018-12) and MerckSerono China Research Fund for Fertility Experts.

\section{Publisher's Note}

Springer Nature remains neutral with regard to jurisdictional claims in published maps and institutional affiliations.

Received: 20 October 2017 Accepted: 20 April 2018

Published online: 30 April 2018

\section{References}

1. Anger JT, Gilbert BR, Goldstein M. Cryopreservation of sperm: indications, methods and results. J Urol. 2003;170(4 Pt 1):1079-84.

2. Hourvitz A, Goldschlag DE, Davis OK, Gosden LV, Palermo GD, Rosenwaks Z. Intracytoplasmic sperm injection (ICSI) using cryopreserved sperm from men with malignant neoplasm yields high pregnancy rates. Fertil Steril. 2008;90(3):557-63.

3. Sanger WG, Olson JH, Sherman JK. Semen cryobanking for men with cancer-criteria change. Fertil Steril. 1992;58(5):1024-7.

4. van Casteren NJ, van Santbrink EJ, van Inzen W, Romijn JC, Dohle GR. Use rate and assisted reproduction technologies outcome of cryopreserved semen from 629 cancer patients. Fertil Steril. 2008;90(6):2245-50.

5. Mack SR, Zaneveld LJ. Acrosomal enzymes and ultrastructure of unfrozen and cryotreated human spermatozoa. Gamete Res. 1987;18(4):375-83.

6. McLaughlin EA, Ford WC, Hull MG. Motility characteristics and membrane integrity of cryopreserved human spermatozoa. J Reprod Fertil. 1992;95(2):527-34.

7. Alvarez JG, Storey BT. Evidence that membrane stress contributes more than lipid peroxidation to sublethal cryodamage in cryopreserved human sperm: glycerol and other polyols as sole cryoprotectant. J Androl. 1993;14(3):199-209.

8. Lassalle B, Testart J. Human zona pellucida recognition associated with removal of sialic acid from human sperm surface. J Reprod Fertil. 1994;101(3):703-11.

9. Benoff S. Carbohydrates and fertilization: an overview. Mol Hum Reprod 1997;3(7):599-637.

10. Centola GM, Raubertas RF, Mattox JH. Cryopreservation of human semen. Comparison of cryopreservatives, sources of variability, and prediction of post-thaw survival. J Androl. 1992;13(3):283-8.

11. Aribarg A, Sukcharoen N, Chanprasit $Y$, Ngeamvijawat J. Prediction of post-thaw sperm motility and sperm cryosurvival rate using the prefreeze sperm parameters. J Med Assoc Thai. 1995;78(9):474-80.

12. Harrison RF, Sheppard BL. A comparative study in methods of cryoprotection for human semen. Cryobiology. 1980;17(1):25-32.

13. Cohen J, Felten $P$, Zeilmaker $\mathrm{GH}$. In vitro fertilizing capacity of fresh and cryopreserved human spermatozoa: a comparative study of freezing and thawing procedures. Fertil Steril. 1981;36(3):356-62

14. Chan SY, Craft IL, Chan YM, Leong MK, Leung CK. The hypoosmotic swelling test and cryosurvival of human spermatozoa. Hum Reprod. 1990;5(6):715-8.

15. Lee CY, Lee CT, Wu CH, Hsu CS, Hsu MI. Kruger strict morphology and post-thaw progressive motility in cryopreserved human spermatozoa. Andrologia. 2012;44(Suppl 1):81-6.
16. Schroter S, Osterhoff C, McArdle W, Ivell R. The glycocalyx of the sperm surface. Hum Reprod Update. 1999;5(4):302-13.

17. Tollner TL, Venners SA, Hollox EJ, Yudin Al, Liu X, Tang G, Xing H, Kays RJ, Lau T, Overstreet JW, Xu X, Bevins CL, Cherr GN. A common mutation in the defensin defb126 causes impaired sperm function and subfertility. Sci Transl Med. 2011;3(92):92ra65

18. Tollner TL, Bevins CL, Cherr GN. Multifunctional glycoprotein defb126 - a curious story of defensin-clad spermatozoa. Nat Rev Urol. 2012:9(7):365-75.

19. Cross NL, Overstreet JW. Glycoconjugates of the human sperm surface: distribution and alterations that accompany capacitation in vitro. Gamete Res. 1987;16(1):23-35.

20. Chandra A, Srinivasan KR, Jamal F, Mehrotra PK, Singh RL, Srivastav A. Post-translational modifications in glycosylation status during epididymal passage and significance in fertility of a $33 \mathrm{kDa}$ glycoprotein (mef3) of rhesus monkey (Macaca mulatta). Reproduction. 2008;135(6):761-70.

21. Purohit S, Laloraya M, Kumar PG. Distribution of $n$ - and o-linked oligosaccharides on surface of spermatozoa from normal and infertile subjects. Andrologia. 2008;40(1):7-12.

22. Xin A, Cheng L, Diao H, Wu Y, Zhou S, Shi C, Sun Y, Wang P, Duan S, Zheng J, Wu B, Yuan Y, Gu Y, Chen G, Sun X, Shi H, Tao S, Zhang Y. Lectin binding of human sperm associates with defb126 mutation and serves as a potential biomarker for subfertility. Sci Rep. 2016;6:20249.

23. Pelaez J, Bongalhardo DC, Long JA. Characterizing the glycocalyx of poultry spermatozoa: iii. Semen cryopreservation methods alter the carbohydrate component of rooster sperm membrane glycoconjugates. Poult Sci. 2011:90(2):435-43.

24. Long JA. Avian semen cryopreservation: what are the biological challenges? Poult Sci. 2006;85(2):232-6.

25. Xin AJ, Cheng L, Diao H, Wang P, Gu YH, Wu B, Wu YC, Chen GW, Zhou SM, Guo SJ, Shi HJ, Tao SC. Comprehensive profiling of accessible surface glycans of mammalian sperm using a lectin microarray. Clin Proteom. 2014;11(1):10.

26. Feng $L$, Ying $M$, Feng $W$, Yong $L$, Ruyao W, Hui L, Wenbo S, Zheng L. Effects of vapor nitrogen freezing method and direct liquid nitrogen freezing method on motility, morphology and ultrastructure of human sperm. J Shanghai Jiaotong Univ. 2012;32(8):6.

27. Tao SC, Li Y, Zhou J, Qian J, Schnaar RL, Zhang Y, Goldstein IJ, Zhu H, Schneck JP. Lectin microarrays identify cell-specific and functionally significant cell surface glycan markers. Glycobiology. 2008;18(10):761-9.

28. Ma F, Wu D, Deng L, Secrest P, Zhao J, Varki N, Lindheim S, Gagneux P. Sialidases on mammalian sperm mediate deciduous sialylation during capacitation. J Biol Chem. 2012;287(45):38073-9.

29. Simon P, Baumner S, Busch O, Rohrich R, Kaese M, Richterich P, Wehrend A, Muller K, Gerardy-Schahn R, Muhlenhoff M, Geyer H, Geyer R, Middendorff R, Galuska SP. Polysialic acid is present in mammalian semen as a post-translational modification of the neural cell adhesion molecule NCAM and the polysialyltransferase st8siaii. J Biol Chem. 2013:288(26):18825-33.

30. Tecle E, Gagneux P. Sugar-coated sperm: unraveling the functions of the mammalian sperm glycocalyx. Mol Reprod Dev. 2015;82(9):635-50.

31. Wu YC, Xin AJ, Lu H, Diao H, Cheng L, Gu YH, Wu B, Tao SC, Li Z, Shi HJ, Zhang YL. Effects of cryopreservation on human sperm glycocalyx. Reprod Contracept. 2017;37(4):6

32. Vilagran I, Yeste M, Sancho S, Castillo J, Oliva R, Bonet S. Comparative analysis of boar seminal plasma proteome from different freezability ejaculates and identification of fibronectin 1 as sperm freezability marker. Andrology. 2015;3(2):345-56.

33. Guimaraes DB, Barros TB, van Tilburg MF, Martins JAM, Moura AA, Moreno FB, Monteiro-Moreira AC, Moreira RA, Toniolli R. Sperm membrane proteins associated with the boar semen cryopreservation. Anim Reprod Sci. 2017:183:27-38

34. Zalazar L, Ledesma A, Hozbor F, Cesari A. Heterologous recombinant protein with decapacitating activity prevents and reverts cryodamage in ram sperm: an emerging biotechnological tool for cryobiology. Anim Reprod Sci. 2016;164:31-9.

35. Rego JP, Martins JM, Wolf CA, van Tilburg M, Moreno F, Monteiro-Moreira AC, Moreira RA, Santos DO, Moura AA. Proteomic analysis of seminal plasma and sperm cells and their associations with semen freezability in guzerat bulls. J Anim Sci. 2016;94(12):5308-20. 
36. Zhang XZ, Xiong CL. Proteins in sperm and seminal plasma associated with human sperm resistance to cryopreservation. Zhonghua Nan Ke Xue. 2013;19(3):214-7.

37. Jiang XP, Wang SQ, Wang W, Xu Y, Xu Z, Tang JY, Sun HY, Wang ZJ, Zhang W. Enolase1 (eno1) and glucose-6-phosphate isomerase (gpi) are good markers to predict human sperm freezability. Cryobiology. 2015;71(1):141-5

38. White T, Bennett EP, Takio K, Sorensen T, Bonding N, Clausen H. Purification and cdna cloning of a human udp-n-acetyl-alpha-d-galactosamine: polypeptide n-acetylgalactosaminyltransferase. J Biol Chem. 1995;270(41):24156-65.

39. Kato K, Takeuchi $\mathrm{H}$, Kanoh A, Mandel U, Hassan H, Clausen H, Irimura T. $\mathrm{N}$-acetylgalactosamine incorporation into a peptide containing consecutive threonine residues by udp-n-acetyl-d-galactosaminide: polypeptide n-acetylgalactosaminyltransferases. Glycobiology. 2001;11(10):821-9.

40. Froman DP, Engel HN Jr. Alteration of the spermatozoal glycocalyx and its effect on duration of fertility in the fowl (Gallus domesticus). Biol Reprod. 1989:40(3):615-21.
Ready to submit your research? Choose BMC and benefit from:

- fast, convenient online submission

- thorough peer review by experienced researchers in your field

- rapid publication on acceptance

- support for research data, including large and complex data types

- gold Open Access which fosters wider collaboration and increased citations

- maximum visibility for your research: over $100 \mathrm{M}$ website views per year

At BMC, research is always in progress.

Learn more biomedcentral.com/submissions 